\section{A4.2 ADIPOCYTES MODULATE T CELL FUNCTION THROUGH RELEASE OF LIPIDS}

doi:10.1136/annrheumdis-2013-203217.2

${ }^{1}$ Andreea loan-Facsinay, ' Joanneke C Kwekkeboom, 'Sanne Westhoff, ${ }^{2}$ Martin Giera, ${ }^{1} Y o a n n$ Rombouts, 'Tom WJ Huizinga, ${ }^{2}$ André Deelder, 'Margreet Kloppenburg, 'René EM Toes. 'Dept. of Rheumatology, Leiden University Medical Center, Leiden, The Netherlands; ${ }^{2 B}$ Biomolecular Mass Spectrometry Unit, Department of Parasitology, Leiden University Medical Center, Leiden, The Netherlands

Background and Objectives Obesity is characterised by the presence of inflammation in adipose tissue. Accumulation of several immune cell-types, including CD4+ T cells, has been previously reported in the increasing adipose tissue. This accumulation is also paralleled by changes in cytokine profiles and phenotype of the infiltrating cells. One of the possible mechanisms involved in these changes is the modulation of $\mathrm{T}$ cell function by tissue-resident adipocytes. Therefore, we investigated whether adipocytes derived from various adipose tissues can modulate CD4+ T cell cytokine production and proliferation and studied the mechanisms involved in this process.

Materials and Methods CD4+ T cells were purified from peripheral blood mononuclear cells using magnetic beads coated with anti-human CD4. Plate-bound anti-CD3 and soluble anti-CD28 antibodies were used to activate $\mathrm{T}$ cells. Adipocytes were isolated from IFP of OA patients by collagenase digestion and were either cultured with purified CD4+ T cells or were cultured in vitro for 24 hours in DMEM/F12 medium supplemented with 0.5\% bovine serum albumin to generate adipocyte-conditioned medium (ACM). Cytokine/adipokine production was measured by intracellular cytokine staining (ICS), ELISA or cytokine multiplex. Lipids were isolated using hapten and lipid profiling was performed by liquid chromatography combined with mass spectrometry.

Results CD4 $+\mathrm{T}$ cells produced increased levels of IFN $\gamma$ when activated in the presence of adipocytes. This effect is mediated by soluble mediators, as shown in transwell and adipocyte-conditioned medium (ACM) transfer experiments. Additionally, ACM induced increased proliferation of CD4 $+\mathrm{T}$ cells upon activation. Furthermore, adipose tissue contained more IFN $\gamma$-producing CD4 $+\mathrm{T}$ cells than peripheral blood of the same individuals, in 3 out of 3 cases tested, which indicates a possible in vivo relevance of our results. To investigate the possible molecular mechanisms involved in this effect, we separated the protein and lipid fraction of ACM. Surprisingly, despite previous data indicating that several adipocyte-derived proteins can modulate $\mathrm{T}$ cell function, we have found that the increased proliferation of $\mathrm{T}$ cells is mainly due to the lipids isolated from ACM. Further separation of these lipids based on polarity revealed that the modulatory effect is mainly confined to fractions containing free fatty acids. All identified fatty acids were able to individually enhance $\mathrm{T}$ cell proliferation.

Conclusions These data indicate that adipocytes can modulate $\mathrm{CD} 4+\mathrm{T}$ cell function through release of soluble mediators. Remarkably, within the soluble mediators identified, lipids and especially free fatty acids are the most prominent modulators of $\mathrm{T}$ cell proliferation.

\section{A4.3 ADIPOCYTES MODULATE THE PHENOTYPE OF MACROPHAGES THROUGH SECRETED LIPIDS}

doi:10.1136/annrheumdis-2013-203217.3

${ }^{1} \mathrm{IR}$ Klein-Wieringa, 'SN Andersen, 'JC Kwekkeboom, ${ }^{2} \mathrm{M}$ Giera, 'BJE de LangeBrokaar, ${ }^{3} \mathrm{GJVM}$ van Osch, ${ }^{4} \mathrm{AM}$ Zuurmond, ${ }^{5} \mathrm{~V}$ Stojanovic-Susulic, ${ }^{6} \mathrm{RGHH}$ Nelissen, ${ }^{1} \mathrm{TWJ}$ Huizinga, ${ }^{1} \mathrm{M}$ Kloppenburg, ${ }^{1} \mathrm{REM}$ Toes, ${ }^{1} \mathrm{~A}$ loan-Facsinay. ${ }^{1}$ Dept. of Rheumatology, Leiden University Medical Center, Leiden, The Netherlands; ${ }^{2}$ Biomolecular Mass Spectrometry Unit, Department of Parasitology, Leiden University Medical Center, Leiden, The Netherlands; ${ }^{3}$ Dept. of Orthopaedics and Dept. of Otorhinolaryngology, Erasmus MC, University Medical Center, Rotterdam, The Netherlands; ${ }^{4}$ TNO Leiden; The Netherlands; ${ }^{5}$ Janssen Research \& Development, LLC Spring House, Pennsylvania, USA; ${ }^{6}$ Dept. of Orthopaedic Surgery, Leiden University Medical Center, Leiden, The Netherlands
Background Adipose tissue secretes a wide range of soluble factors that can influence whole body metabolism. Previous studies have shown an accumulation of macrophages and an enhanced proinflammatory profile of these cells in adipose tissue of obese mice. Modulation of macrophages by soluble mediators released by adipocytes has been proposed as a possible mechanism underlying these changes. In humans, an increased number of macrophages in adipose tissue of obese individuals have been observed, although no clear change in macrophages phenotype could be established. Moreover, no information exists about the interaction between macrophages and adipocytes in humans.

Objective In the present study, we explored the possibility that adipocytes modulate the phenotype of macrophages and studied the possible molecular pathways involved in this modulation.

Results Treatment of macrophages with adipocyte-conditioned medium (ACM) resulted in a strong reduction in IL-12p40 secretion upon LPS stimulation, whereas TNF $\alpha$ and other cytokines remained largely unaffected. This effect was independent of the source of ACM. Interestingly, the inhibition increased with increase in Body Mass Index (BMI) of the adipocyte donor. Therefore, it was hypothesised that the effect is mediated by a soluble factor whose release is correlated to the BMI of the adipocyte donor. To this end, we measured several cytokines, adipokines and lipids present in ACM. Among these, the release of several free fatty acids (FA) and $\mathrm{PGE}_{2}$ correlated with the BMI of the adipocyte donor. Further tests indicated that oleic and linoleic acid, as well as PGE were able to inhibit IL12p40 secretion, whereas palmitic acid could not. Upon separation of ACM protein and lipid fractions, we confirmed that inhibition of IL12p40 resides mainly in the ACM lipid fraction.

Conclusions These results provide first evidence that obesityrelated changes in macrophage phenotype could be mediated by adipocytes in humans. These effects are mainly mediated through lipids released by adipocytes. Intriguingly, modulation appears different than in murine obesity, indicating that the immunomodulatory effects of obesity could be different in humans and mice.

\section{A4.4 CIRCULATING METASTASIS PROMOTING PROTEIN S100A4 IN IDIOPATHIC INFLAMMATORY MYOPATHIES}

doi:10.1136/annrheumdis-2013-203217.4

'L Pleštilová, ${ }^{2} \mathrm{O}$ Pecha, ${ }^{1} \mathrm{H}$ Mann, 'LA Cerezo, 'J Vencovský, 'L Šenolt. 'Institute of Rheumatology and Department of Rheumatology, $1^{\text {st }}$ Faculty of Medicine, Charles University, Prague; ${ }^{2}$ nstitute of Biophysics and Informatics, $1^{\text {st }}$ Faculty of Medicine, Charles University, Prague

Background and Objectives Metastasis promoting protein S100A4 is involved in the pathogenesis of tumours and chronic autoimmune diseases such as rheumatoid arthritis (RA) and psoriasis. We recently described increased expression of S100A4 in inflamed muscle tissue in patients with idiopathic inflammatory myopathies (IIM). Since circulating levels of S100A4 correlate with disease severity in RA patients, we therefore evaluated the association between S100A4 protein and disease activity in patients with IIM and compared S100A4 serum levels in myositis patients and healthy controls in this study.

Methods Serum levels of S100A4 protein were determined in 43 dermatomyositis (DM), 39 polymyositis (PM) and 22 cancer associated myositis (CAM) patients and in 89 healthy controls. In 11 patients (4 DM, 3 PM, 4 CAM), S100A4 serum levels were measured before and after the start of treatment. The associations between S100A4 levels, inflammation, disease activity and muscle strength were examined. Disease activity was assessed using Disease Activity Core Set Measures developed by International Myositis Assessment \& Clinical Studies Group (IMACS). Serum levels of 
C-reactive protein (CRP) and muscle-associated enzymes creatine phosphokinase (CK) and lactate dehydrogenase (LD) were measured by routine laboratory methods.

Results In patients with PM, serum levels of S100A4 protein were significantly higher than those observed in healthy controls or DM patients $(148.6 \pm 351.5$ versus $80.75 \pm 285.1, p<0.01$ and $43.55 \pm$ $53.03, \mathrm{p}<0.05$, respectively). No significant differences in S100A4 levels were found between CAM patients $(119.9 \pm 414.0)$ and healthy controls or other myositis patients. In the whole group of IIM patients, serum S100A4 levels correlated with MYOACT score $(\mathrm{r}=0.39 ; \mathrm{p}<0.001)$ and its components Constitutional Disease Activity (DA) $(r=0.34 ; \mathrm{p}<0.001)$ and Pulmonary DA $(r=0.44$; $p<0.001)$. Serum S100A4 correlated also with Muscular DA $(r=0.25 ; p<0.05)$, CK $(r=0.33 ; p<0.01)$ and LD $(r=0.40$; $p<0.001)$. S100A4 levels correlated with Cutaneous DA ( $r=0.46$; $\mathrm{p}<0.01$ ) in DM patients and with Extramuscular Global Assessment only in PM patients $(r=0.55 ; \mathrm{p}<0.001)$. No significant correlations of S100A4 serum levels in patients with CAM were found. In the 11 longitudinal IIM samples there was no significant decrease of S100A4 serum levels observed. Multiple regression of the whole IIM patients group showed significant association of S100A4 serum levels with Pulmonary DA $(\beta=0.369 ; p<0.01)$, LD $(\beta=0.345$; $p<0.01)$ and severity of dysphagia $(\beta=-0.250 ; p<0.05)$. In $P M$ patients, S100A4 levels were associated with Extramuscular Global Assessment $(\beta=0.552 ; \mathrm{p}<0.01)$ and in DM patients with MYOACT $(\beta=0.557 ; p<0.01)$ and $\operatorname{CRP}(\beta=0.391 ; p<0.05)$.

Conclusions This is the first study showing that circulating levels of S100A4 are associated with several features of IIM disease activity, particularly with extramuscular components. We did not find any association of S100A4 levels and cancer associated myositis. Further studies analysing bioactive form of S100A4 and the role of S100A4 in cancer associated myositis are needed.

\section{A4.5 DO HIGH MOLECULAR WEIGHT ADIPONECTIN LEVELS ASSOCIATE WITH RADIOGRAPHIC PROGRESSION IN EARLY RHEUMATOID ARTHRITIS AND HAND OSTEOARTHRITIS?}

doi:10.1136/annrheumdis-2013-203217.5

IIR Klein-Wieringa, 'SN Andersen, 'JC Kwekkeboom, 'L Herb-v.Toorn, 'AHM van der Helm-van Mil, $2 \mid$ Meulenbelt, 'TWJ Huizinga, 'M Kloppenburg, 'REM Toes, ${ }^{1}$ A loan-Facsinay. 'Dept. of Rheumatology, Leiden University Medical Center, Leiden, The Netherlands; ${ }^{2}$ Dept. of Molecular Epidemiology, Leiden, The Netherlands

Background Adipose tissue can secrete many different soluble factors (adipokines) influencing whole body metabolism. Some of these adipokines, such as adiponectin, have been shown to influence radiographic progression in osteoarthritis $(O A)$ and rheumatoid arthritis (RA). In RA patients, total adiponectin (totAPN) levels in serum associate positively with radiographic progression, which suggestes an adverse effect on disease. Intruiguingly, in patients with hand OA, high totAPN levels in serum associated with a decreased relative risk for radiographic progression, suggesting a benificial effect on disease.

Adiponectin is a pleiotropic adipokine, which consists of several isoforms. Of these isoforms, high molecular weight adiponectin (hmwAPN) has been described as one of the most biologically active and its effect on radiographic progression in $\mathrm{RA}$ and hand $\mathrm{OA}$ is unknown.

Objective Therefore, we explored the possibility that the association between totAPN and disease progression is primarily mediated by the hmwAPN isoform.

Methods Concentrations of hmwAPN and totAPN were determined in baseline plasma of 324 RA patients from the Early Arthritis Cohort (EAC) and in baseline sera of 164 hand OA patients from the Genetics Arthrosis and Progression (GARP) study. The association between levels of hmwAPN and totAPN with radiographic progression were determined using a multivariate normal regression model (EAC cohort) or by generalised estimated equations (GARP cohort). Adjustments were made for age, gender, treatment strategy and Body Mass Index (BMI).

Results In RA patients totAPN associated positively with radiographic progression (Sharp van der Heijde scores) (association estimate $3.65, p=0.002$ ), whereas in patients with hand OA, totAPN associated negatively with radiographic progression (joint space narrowing (JSN)) (Odds 0.24/Odds 0.21, $p=0.002 / p=0.002$ two highest tertiles compared to the lowest tertile). HmwAPN on the other hand, did not associate significantly with radiographic progression in patients with hand OA or RA, although in patients with RA we did observe a trend towards a positive association (association estimate $1.53 p=0.07$ ) upon correcting for age, gender and treatment strategy. This trend was lost after further adjustment for BMI. Similar results were obtained when joint space narrowing (JSN) was used as outcome measurement.

Conclusions Our data further substantiate the connexion between APN-levels and radiographic progression in rheumatic disease and indicate that the differential effects associated between totAPN and radiographic progression in either in $\mathrm{RA}$ and hand $\mathrm{OA}$ is not mediated by (a selective effect of) hmwAPN.

\section{A4.6 EFFECTS OF CHOLIC ACID AND ITS DERIVATIVES IN EXPERIMENTAL ARTHRITIS}

doi:10.1136/annrheumdis-2013-203217.6

'Silvia Hayer, ${ }^{2}$ Emina Halilbasic, 'Birgit Niederreiter, 'Martin Willburger, 'Peter Mandl, 'Victoria Saferding, 'Stephan Blüml, 'Josef Smolen, ${ }^{2}$ Michael Trauner, 'Kurt Redlich. 'Medical University of Vienna, Department of Internal Medicine III, Division of Rheumatology; ${ }^{2}$ Medical University of Vienna, Department of Internal Medicine III, Division of Gasteroenterology

Introduction Bile acids play an important role in cholesterol metabolism and act as intestinal detergents for digestion and absorption of fats and fat-soluble vitamins. Disruption of bile flow causes cholestatic liver diseases. Derivatives of cholic acid (CA) such as nor-ursodeoxycholic acid (norUDCA) are promising therapeutic agents in the treatment of cholangiopathies. Previous studies also demonstrated anti-inflammatory and anti-fibrotic properties of norUDCA in experimental sclerosing cholestasis.

Objective To investigate the anti-inflammatory potential of CA and its derivatives ursodeoxycholic acid (UDCA) and nor-UDCA in in Collagen-induced arthritis (CIA), an animal model for inflammatory, erosive arthritis.

Methods Mice were prophylactically treated with CA, UDCA or nor-UDCA enriched diet pellets $(5 \mathrm{mg} / \mathrm{kg}$ diet $)$ or standard diet pellets (Placebo) ad libitum starting 1 week before the first immunisation with collagen. Animals were weekly assessed for clinical signs of arthritis, body weight and food consumption during the experimental period. After 10 weeks of treatment hind paws, liver, sera and lymph nodes were isolated for further analysis. Sera were investigated for anti-collagen antibodies, cytokine responses and liver parameters such as alkaline phosphatase (AP) and alanine transaminase (ALT). Paraffin-sections of hind paws were examined for histoptahological changes in synovial inflammation, subchondral bone erosion, cartilage damage and osteophyte formation. Cell populations within synovial pannus were identified by immunhistochemical stainings and were determined using HistoQuest software (from TissueGnostics).

Results Uptake of CA, UDCA and norUDCA was confirmed by serum analysis. Prophylactic treatment of CIA mice with UDCA and norUDCA could not significantly prevent disease incidence. In contrast, treatment with CA led to a marked increase in disease incidence and severity compared to Placebo treated animals. Whereas UDCA and norUDCA showed a similar course of clinical signs of 